\title{
Postoperative analgesia in patients undergoing robot-assisted thoracic surgery: a comparison between thoracic epidural analgesia and intercostal nerve block combined with intravenous patient-controlled analgesia
}

\author{
Izumi Kawagoe, Masakazu Hayashida, Daizoh Satoh, Tsukasa Kochiyama, Masataka Fukuda, Jun Kishii \\ Department of Anesthesiology and Pain Medicine, Juntendo University School of Medicine, Tokyo, Japan \\ Contributions: (I) Conception and design: I Kawagoe; (II) Administrative support: D Satoh, M Hayashida; (III) Provision of study materials or patients: \\ T Kochiyama, M Fukuda, J Kishii; (IV) Collection and assembly of data: I Kawagoe, T Kochiyama, M Fukuda; (V) Data analysis and interpretation: I \\ Kawagoe; (VI) Manuscript writing: All authors; (VII) Final approval of manuscript: All authors. \\ Correspondence to: Izumi Kawagoe, MD, PhD. Department of Anesthesiology and Pain Medicine, Juntendo University School of Medicine, 2-1-1 \\ Hongo, Bunkyo-ku, Tokyo 113-8421, Japan. Email: ikawago@juntendo.ac.jp.
}

\begin{abstract}
Background: Recently, robot-assisted thoracic surgery (RATS) is increasingly applied to lung or mediastinal tumor surgery. However, appropriate methods of postoperative analgesia for RATS have not been studied.

Methods: Patients who underwent RATS at a single university hospital between January, 2017 and March, 2018 were studied retrospectively. Patients were anesthetized with either general anesthesia alone or combined general and thoracic epidural anesthesia. Accordingly, postoperative analgesia was managed with either intravenous patient-controlled analgesia (PCA) with fentanyl or thoracic epidural analgesia (TEA) with morphine and levobupivacaine. Patients were thus divided into 2 groups (PCA and TEA) according to methods of postoperative analgesia, and analgesic efficacies were compared between the groups with regard to pain scores evaluated on a 11-point numerical rating scale (NRS) at 0, 3, 6, 12, 18, 24, and $48 \mathrm{~h}$ postoperatively, rescue analgesic requirements within $24 \mathrm{~h}$, side effects of anesthesia and analgesia, including respiratory depression, hypotension, nausea, pruritus, and urinary retention, time to ambulation after surgery, and hospital stay after surgery.
\end{abstract}

Results: Data from 107 patients (76 in Group PCA and 31 in Group TEA) were analyzed. NRS pain scores at 6,18 , and $48 \mathrm{~h}$ were significantly less or tended to be less in Group TEA than in Group PCA $(1.8 \pm 2.0 \mathrm{vs}$. $2.6 \pm 1.8, \mathrm{P}=0.045 ; 1.7 \pm 1.5$ vs. $2.4 \pm 1.8, \mathrm{P}=0.047$; and $1.9 \pm 1.4$ vs. $2.5 \pm 1.6, \mathrm{P}=0.063$, respectively). The number of patients who required rescue analgesics within $24 \mathrm{~h}$ was significantly less in Group TEA than in Group PCA [4/31 (12\%) vs. 32/76 (42\%), P=0.004]. The other parameters were not significantly different between the groups.

Conclusions: Compared with PCA, TEA provided better analgesia after RATS in terms of less pain scores, less rescue analgesic requirements, and similar side effect profiles. TEA with a hydrophilic opioid and local anesthetic seemed an appropriate method of postoperative analgesia in patients undergoing RATS.

Keywords: Robot-assisted thoracic surgery (RATS); postoperative analgesia; thoracic epidural analgesia (TEA); intravenous patient-controlled analgesia (PCA); intercostal nerve block

Submitted Aug 13, 2020. Accepted for publication Nov 02, 2020.

doi: 10.21037/apm-20-1607

View this article at: http://dx.doi.org/10.21037/apm-20-1607 


\section{Introduction}

Robot-assisted thoracic surgery (RATS) is a relatively new technique that launched in the early 1990s. RATS has been increasingly applied because of its lesser invasiveness, clearer three-dimensional visualization of the surgical field, more precise dexterity, and easier accessibility to targets with more flexible devices, compared with open or video-assisted thoracic surgery (VATS) $(1,2)$. Its safety and feasibility have been proven in the last decade $(3,4)$. VATS also is recognized to be advantageous over open thoracotomy because of lesser invasiveness $(5,6)$. Compared to open thoracotomy, hospital stay is shorter and postoperative complications is fewer after RATS (7). In addition, emergency conversion to open thoracotomy may be fewer, and mortality may be lower with RATS, compared with VATS $(1,8)$.

However, the number of incisions for surgical ports is not less or even more, and the incisions range over more intercostal spaces in RATS than in VATS $(9,10)$. Further, in contrast to VATS devices inserted perpendicular to the skin via centers of intercostal spaces and operated manually not to touch the ribs, the robot devices are inserted from the caudal ends of intercostal spaces, directed at an approximately $45^{\circ}$ angle cephalically due to the specific arrangement of robot arms, and driven by powerful mechanical forces $(11,12)$. Therefore, robot devices may touch or even compress ribs bordering each intercostal space. Actually, we have experienced a case of suspected rib injuries associated with a RATS procedure. Although RATS is considered minimally invasive, it remains to be proven whether RATS is actually a minimal invasive procedure, compared with conventional VATS, and further, compared with recently introduced uniportal VATS (13). Postoperative pain after RATS thus may not be minimal, or even be more intense than expected.

To our knowledge, data are currently unavailable on what method of analgesia is appropriate for postoperative pain control after RATS, although recent studies have compared intensity of postoperative pain among surgical procedures including RATS $(14,15)$. In the present study, we compared efficacies of postoperative pain control after RATS between intercostal nerve block combined with i.v. patient-controlled analgesia (PCA) and thoracic epidural analgesia (TEA).

We present the following article in accordance with the TREND reporting checklist (available at http://dx.doi. org/10.21037/apm-20-1607).

\section{Methods}

The study was conducted in accordance with the Declaration of Helsinki (as revised in 2013). The study was approved by institutional ethics board of Juntendo University Hospital (No. 18-316) and individual consent for this retrospective analysis was waived.

\section{Patients}

A total of 111 consecutive patients who were scheduled for RATS at our institution between January, 2017 and March, 2018 were studied. Anesthesia was performed either with general anesthesia alone or with combined general and thoracic epidural anesthesia at the discretion of anesthesiologists and surgeons in charge of each anesthesia and surgery. Accordingly, postoperative analgesia was provided either by a combination of intercostal nerve block (ICB) and i.v. patientcontrolled analgesia (PCA) or by thoracic epidural analgesia (TEA), as mentioned below. Patients were thus divided into 2 groups, Group PCA and Group TEA, depending on the primary methods of postoperative analgesia, and efficacies of postoperative analgesia were compared between the groups.

\section{Anesthesia and postoperative analgesia in Group PCA}

In patients in Group PCA, general anesthesia was induced and maintained with total intravenous anesthesia using target-controlled infusion of propofol $(1.5-3 \mu \mathrm{g} / \mathrm{mL})$ and continuous infusion of remifentanil $(0.1-0.4 \mu \mathrm{g} / \mathrm{kg} / \mathrm{min})$. Doses of these drugs were adjusted so as to maintain the bispectral index (BIS) between 40 and 60 and to achieve stable hemodynamics during anesthesia and surgery. Rocuronium was intermittently given to achieve muscle relaxation for tracheal intubation and surgical procedures. Fentanyl was given as necessary. One-lung ventilation (OLV) was achieved with a standard left-sided double-lumen tube. At the beginning of surgery, surgeons performed 4 to 6 ICBs with $0.25 \%$ levobupivacaine $(3 \mathrm{~mL})$ for each ICB at each incision site. A disposable PCA pump (Coopdech Syrinjector PCA Set ${ }^{\circledR}$, Daiken Medical, Tokyo, Japan), in which a continuous infusion rate, bolus dose, lockout time, and hourly limit were set at $1 \mathrm{~mL} / \mathrm{h}, 1 \mathrm{~mL}, 10 \mathrm{~min}$, and $7 \mathrm{~mL}$, respectively, were filled with fentanyl $(1,000 \mu \mathrm{g}$ in $20 \mathrm{~mL}$ ) and normal saline $(20 \mathrm{~mL}$ ) (final fentanyl concentration, $25 \mu \mathrm{g} / \mathrm{mL}$ ), allowing to deliver up to $175 \mu \mathrm{g} / \mathrm{h}$ of fentanyl. Droperidol (2.5-5 $\mathrm{mg})$ was added or not added to the infuscate at the discretion of 
anesthesiologists, although reportedly, its addition reduces postoperative nausea and vomiting, and supports the continuation of i.v. PCA with fentanyl (16). The infusion of fentanyl was started at the end of surgery, following incremental bolus doses of fentanyl to achieve adequate immediate postoperative analgesia. Infusion of fentanyl with the PCA pump was continued until postoperative day (POD) 1 or 2.

\section{Anesthesia and postoperative analgesia in Group TEA}

In patients in Group TEA, an epidural catheter was inserted via a mid-thoracic intervertebral space using a loss of resistance technique with normal saline. The effect of epidural analgesia was confirmed with loss of cold sensation $5 \mathrm{~min}$ after injection of $2 \%$ lidocaine $(2 \mathrm{~mL})$. Then, general anesthesia was induced and maintained with total intravenous anesthesia using propofol and remifentanil, as mentioned above. Rocuronium was given for muscle relaxation required for tracheal intubation and surgery. Fentanyl was given as necessary. Before surgery, a combination of $0.25 \%$ levobupivacaine $(4 \mathrm{~mL})$, fentanyl (50 $\mu \mathrm{g})$, and morphine (1-2 $\mathrm{mg}$ ) was injected into the epidural space. A disposable infusion pump with a PCA function (bolus dose, $3 \mathrm{~mL}$; and lockout time, $30 \mathrm{~min}$ ) (Rakuraku Fusor ${ }^{\circledR}$, Smiths Medical Japan, Tokyo, Japan) was filled with $0.25 \%$ levobupivacaine $(144 \mathrm{~mL})$ and morphine (2-6 mg). Droperidol (2.5-5 mg) was added or not added to the infusate at the discretion of anesthesiologists, although reportedly, its addition inhibits pruritus, nausea, and vomiting during epidural morphine analgesia (17). Continuous epidural infusion for intra- and post-operative analgesia was started at a rate of $3 \mathrm{~mL} / \mathrm{h}$ during surgery. The infusion was continued until POD 1 or 2 .

\section{Surgical procedures}

Robotic surgery using the da Vinci surgical system (Intuitive Surgical, Sunnyvale, CA, USA) was performed with a three or four-arm technique via incisions placed between the third and ninth intercostal spaces depending on the procedures. One or two additional VATS camera ports were inserted via almost the same intercostal spaces as the Robot arms. The patients were placed in the lateral jack-knife position for lung resection and in the supine position for anterior mediastinal tumor resection. In addition to OLV, artificial pneumothorax at a pressure of 5 to $12 \mathrm{mmHg}$ was achieved with $\mathrm{CO}_{2}$ insufflation. After completion of the procedure, one or two chest drains were placed via the most dorsal port. The drains were removed when air fistulation was absent and drainage volume was below $300 \mathrm{~mL} / 24 \mathrm{~h}$.

\section{Collected data}

Perioperative parameters related to demography, anesthesia, surgery, postoperative pain, side effects of anesthesia and analgesia, and clinical outcomes were investigated and compared between Group PCA and Group TEA. Data on doses of fentanyl and remifentanil required during anesthesia, operation time, surgical bleeding, and feasibility of extubation in the operating room were collected from anesthesia records. Data on patients' demography and comorbidities, surgical procedures, intensity of postoperative pain at rest evaluated by the ward nurses on a numerical rating scale (NRS) $(0$ : no pain, 10 : worst pain imaginable) at $0,3,6,12,18,24$, and 48 h postoperatively, rescue analgesics [i.v. pentazocine $(30 \mathrm{mg})$, acetaminophen $(1,000 \mathrm{mg})$, or flurbiprofen $(50 \mathrm{mg})]$ required within $24 \mathrm{~h}$ postoperatively, major side effects (respiratory depression), and minor side effects (hypotension defined as systolic blood pressure $<90 \mathrm{mmHg}$, nausea treated with antiemetics, urinary retention, and pruritus) recorded within $48 \mathrm{~h}$ postoperatively, time to ambulation after surgery, and hospital stay after surgery were collected from medical records.

\section{Statistical analysis}

Statistical analysis was performed with SPSS 25.0 (SPSS, Chicago, IL, USA). All variables are shown by the methods of descriptive statistics [mean and standard deviation (SD) or frequency], as previously described (14). The distribution of variables was examined for normality using the Kolmogorov-Smirnov test. Levebe's test was used to assess the equality of variance in the different samples. In order to test differences between means, unpaired $t$-test was applied in case of normal distribution; otherwise, the MannWhitney U-test was used. Categorical data were compared with Fisher's probability test or chi-square test. Intra-group differences were tested with paired $t$-test or Wilcoxon signed rank test with Bonferroni correction, as appropriate. A value of $\mathrm{P}<0.05$ was considered statistically significant. A sample size calculation to identify a 1.0 difference in the NRS scores based on the SD value of 1.3 reported in a previous study indicated that 27 patients would be required per group, given that $\alpha$ is 0.05 with a two-sided test and a 


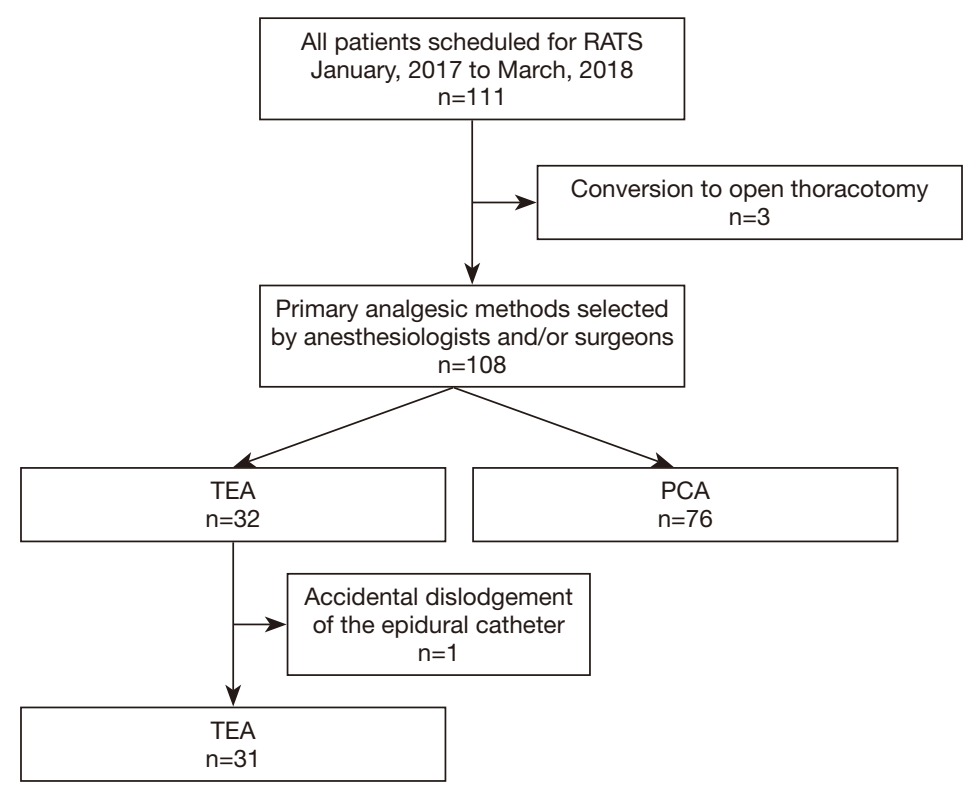

Figure 1 Flow diagram of the study. TEA, thoracic epidural analgesia; PCA, patient-controlled analgesia.

power of $0.8(14)$.

\section{Results}

A total of 111 patients were scheduled for RATS during the study period. Because RATS was converted to open thoracotomy in three patients, 108 patients were included in the study. Of them, 76 patients received ICB plus i.v. PCA with fentanyl and 32 received TEA with morphine and levobupivacaine for perioperative pain control. Because the epidural catheter was accidentally dislodged in one patient, however, data from 107 patients (76 in Group PCA and 31 in Group TEA) were analyzed (Figure 1). The number of patients in each group was more than the sample size calculated by the priori power analysis.

All patients in both groups were extubated immediately after surgery in the operating room. Demographic or surgical data were not different between the two groups (Table 1). Doses of fentanyl and remifentanil required during anesthesia were significantly less in Group TEA than in Group PCA (Table 2). The number of patients who required rescue analgesics within $24 \mathrm{~h}$ was significantly less in Group TEA than in Group PCA, although the kinds of rescue analgesics used were not different between the groups (Table 2). The NRS pain scores at 6, 18, and $48 \mathrm{~h}$ were significantly less or tended to be less in Group TEA than in Group PCA (Table 3). The pain scores did not change significantly over time in either group (Table 3). The number of patients who received droperidol in the infuscate was not different between the groups (Table 4). The incidences of side effects, including hypotension and nausea, time to ambulation, and hospital stay after surgery were not significantly different between the groups (Table 4).

\section{Discussion}

The current data demonstrated that TEA with morphine and a local anesthetic provided better postoperative analgesia after RATS, compared with ICB plus i.v. PCA with fentanyl, in terms of less pain scores, less rescue analgesic requirements, and similar side effect profiles. Although a few previous studies have evaluated intensity of postoperative pain after RATS $(14,15)$, our study is the first to compare efficacies of different methods of postoperative analgesia after RATS.

Adequate postoperative analgesia after thoracic surgery is important not only to improve patient's satisfaction but also to reduce the risk of postoperative complications $(15,18,19)$. Further, adequate postoperative analgesia after thoracic surgery may reduce the incidence of chronic postthoracotomy pain (20). TEA and para-vertebral block (PVB) have been recognized to be two major methods of postoperative analgesia not only for open thoracotomy but also for VATS $(18,21,22)$. Although the incidence of minor 
Table 1 Demographic and surgical data

\begin{tabular}{lccc}
\hline Demographic and surgical data & PCA $(\mathrm{n}=76)$ & TEA $(\mathrm{n}=31)$ & $9 / 23$ \\
\hline Sex (males/females) & $23 / 53$ & $62.2[11.3]$ & 0.893 \\
Age (years) & $65.5[12.5]$ & $159.2[7.8]$ & 0.193 \\
Height (cm) & $159.7[9.3]$ & $58.9[11.0]$ & 6.827 \\
Weight $(\mathrm{kg})$ & $59.3[13.8]$ & $6(19 \%)$ & 0.871 \\
Patients with cardiovascular disease & $9(12 \%)$ & $2(6 \%)$ & 0.31 \\
Patients with diabetes mellitus & $10(13 \%)$ & $1(3 \%)$ & 0.319 \\
Patients with cerebrovascular disease & $4(5 \%)$ & $0(0 \%)$ & 0.651 \\
Patients with chronic kidney disease & $2(3 \%)$ & $19 / 7 / 5$ & 0.362 \\
Performed procedures (L/S/M) & $50 / 16 / 10$ & $167[65]$ & 0.639 \\
Operation time (minutes) & $167[57]$ & $16[29]$ & 0.977 \\
Intraoperative bleeding (mL) & $15[19]$ & 0.907 \\
\hline
\end{tabular}

Data are presented as mean [SD] or number (\%). L, lobectomy; S, segmentectomy; M, mediastinal tumor resection.

Table 2 Perioperative medications

\begin{tabular}{|c|c|c|c|}
\hline Perioperative medications & PCA $(n=76)$ & TEA $(n=31)$ & $P$ value \\
\hline Remifentanil required during anesthesia $(\mu \mathrm{g})$ & 304 [169] & 200 [103] & $<0.001$ \\
\hline Patients receiving/not receiving droperidol in infusers & $51 / 25$ & $26 / 5$ & 0.099 \\
\hline Patients requiring rescue analgesics within $24 \mathrm{~h}$ & $32(42 \%)$ & $4(12 \%)$ & 0.004 \\
\hline
\end{tabular}

Data are shown as mean [SD] or number (\%).

Table 3 Pain scores at rest evaluated on an 11-point NRS at $0-48 \mathrm{~h}$ postoperatively

\begin{tabular}{lccc}
\hline Pain scores at rest & Group PCA $(\mathrm{n}=76)$ & Group TEA $(\mathrm{n}=31)$ & P value \\
\hline NRS at $0 \mathrm{~h}$ & $3.1(2.8)$ & $2.7(3.0)$ & 0.449 \\
NRS at $3 \mathrm{~h}$ & $2.5(1.9)$ & $2.0(2.3)$ & 0.291 \\
NRS at $6 \mathrm{~h}$ & $2.6(1.8)$ & $1.8(2.0)$ & 0.045 \\
NRS at $12 \mathrm{~h}$ & $2.3(1.8)$ & $1.9(1.9)$ & 0.214 \\
NRS at $18 \mathrm{~h}$ & $2.4(1.8)$ & $1.7(1.5)$ & 0.047 \\
NRS at $24 \mathrm{~h}$ & $2.3(1.7)$ & $1.9(1.4)$ & 0.250 \\
NRS at $48 \mathrm{~h}$ & $2.5(1.6)$ & $1.9(1.4)$ & 0.063 \\
\hline
\end{tabular}

Data are presented as mean (SD). NRS, numerical rating scale. 
Table 4 Side effects of anesthesia/analgesia and postoperative outcomes

\begin{tabular}{lccc}
\hline Side effects and postoperative outcomes & PCA $(\mathrm{n}=76)$ & TEA $(\mathrm{n}=31)$ & $\mathrm{P}$ value \\
\hline Nausea requiring antiemetics within $48 \mathrm{~h}$ & $7(9 \%)$ & $1(3 \%)$ & 0.607 \\
Hypotension within $48 \mathrm{~h}$ & $0(0 \%)$ & $0(0 \%)$ & 0.126 \\
Pruritus within $48 \mathrm{~h}$ & $1(1.3 \%)$ & $0(0 \%)$ & 0.117 \\
Urinary retention within $48 \mathrm{~h}$ & $0(0 \%)$ & $0(0 \%)$ & - \\
Respiratory depression within $48 \mathrm{~h}$ & $0(0 \%)$ & $1.0[0.2]$ & - \\
Time to ambulation after surgery (days) & $1.0[0.2]$ & $4.5[1.7]$ & 0.837 \\
Hospital stay after surgery (days) & $4.4[1.3]$ & & 0.916 \\
\hline
\end{tabular}

Data are presented as mean (SD) or number (\%).

side effects such as perioperative hypotension is less with PVB than with TEA, analgesic efficiencies, incidence of major side effects such as hematoma, and patients' outcomes are considered comparable between TEA and PVB (21-23).

Recently, the number of RATS is increasing, while that of open thoracotomies is decreasing, and that of VATS remains unchanged (7). Three-dimensional visualization, 10x magnified view, shake reduction, and small-wristed instruments provide high-quality surgical procedures in RATS $(1,2,24)$. However, there are some disadvantages of RATS, such as less time- and cost-effective performances (24). Further, postoperative pain after RATS may be more intense than expected because of surgical incisions ranging over many intercostal spaces and quite unique operating modes of devices in RATS in contrast to VATS, as mentioned above (9-13).

Although RATS is increasingly applied, only a few studies have addressed acute postoperative pain after RATS. Recent studies comparing postoperative pain among different surgical procedures showed that acute postoperative pain after RATS remained as intense as that after open thoracotomy until POD 3, although it became less intense on POD 4 or later, compared with open thoracotomy $(14,15)$. Moreover, the number of patients who felt that the approach affected pain was significantly more after RATS than after VATS, and the incidences of postoperative chronic neuropathic pain was $34.6 \%$ after RATS, compared to $19.8 \%$ after VATS (15). These previous findings suggest that the tissue injuries due to RATS and intensity of acute pain during the early postoperative period after RATS can be considerably severe, as suggested also by the present data showing higher pain scores and more rescue analgesic requirements in Group PCA than in Group TEA. These previous and present data suggest that the intensive postoperative analgesic methods used for open thoracotomy such as TEA and PVB may be appropriate also to control pain following RATS at least during the early postoperative period.

Because the artificial pneumothorax and the extreme lateral jack-knife position required for RATS can occasionally induce severe intraoperative hypotension (25), it would be desirable to select an analgesic method that affects hemodynamics less significantly. In this regard, TEA with a hydrophilic opioid, which can reduce the local anesthetic dose requirement while enhancing the spread of analgesia, seems superior to TEA with a local anesthetic alone because this can reduce the risk of perioperative hypotension (26). Further, TEA with a hydrophilic opioid and local anesthetic provides enhanced analgesia over TEA or continuous PVB with a local anesthetic alone (26). Actually, in the present study, epidural infusion of $0.25 \%$ levobupivacaine containing low-dose morphine at $3 \mathrm{~mL} / \mathrm{h}$ rarely induced postoperative hypotension while providing enhanced analgesia over i.v. PCA with fentanyl. Therefore, TEA with a hydrophilic opioid and local anesthetic seems to be one of the appropriate choices for pain control after RATS.

We used morphine for epidural analgesia, and fentanyl for i.v. PCA. Probably, results would have been different with a different opioid used for each route. However, we used epidural morphine because when given epidurally, hydrophilic morphine results in a wider spread of analgesia, compared with lipophilic fentanyl (26-28). Conversely, we selected fentanyl for i.v. PCA because lipophilic fentanyl has faster onset of analgesic action suitable for acute pain control, compared with hydrophilic morphine, even though its shorter duration of action may demand more frequent self-administration to maintain 
adequate analgesia (29).

Many kinds of blocks including TEA and PVB are employed as a part of multimodal analgesia (18). We used preoperative ICB plus postoperative i.v. PCA with fentanyl in patients without thoracic epidural catheters. However, significantly more doses of remifentanil and fentanyl required during anesthesia in Group PCA than in Group TEA observed in the present study indicated that ICB was much less effective in providing intraoperative analgesia, compared with TEA. Further, ICB performed preoperatively possibly did not contribute to postoperative analgesia, given its relatively short duration of action (30). The present data in RATS patients seemed to be in good agreement with previous data showing that ICB performed at the end of surgery plus i.v. PCA with morphine was inferior to TEA with respect to pain control in patients undergoing open thoracotomy (19).

There were some limitations of the present study. First, because of the retrospective study fashion, there might be some limitations in collecting correct and/or important data. Second, we used a disposable PCA pump, in which the settings of an infusion rate, bolus dose, and lockout time were fixed. However, we expected that the setting allowing to deliver up to $175 \mu \mathrm{g} / \mathrm{h}$ of fentanyl would be sufficient to achieve pain relief even in patients with severe postoperative pain. Clearly, a prospective randomized study to compare analgesic efficacies of various methods of postoperative analgesia is needed to identify the most appropriate method of analgesia for pain control after RATS. In the further study, a PCA pump allowing for a more flexible dose delivery should be used so that it could deliver sufficient doses on demand and minimize rescue analgesic requirements, thus simplifying the study design.

\section{Conclusions}

In conclusion, compared with intercostal nerve blocks plus i.v. PCA with fentanyl, TEA with morphine and levobupivacaine provided better postoperative analgesia after RATS. TEA with a hydrophilic opioid and local anesthetic seems to be an appropriate method of postoperative analgesia in patients undergoing RATS.

\section{Acknowledgments}

We thank all the staff contributing to the practice of robotassisted thoracic surgery.

Funding: None.

\section{Footnote}

Reporting Checklist: The authors have completed the TREND reporting checklist. Available at http://dx.doi. org/10.21037/apm-20-1607

Data Sharing Statement: Available at http://dx.doi. org/10.21037/apm-20-1607

Peer Review File: Available at http://dx.doi.org/10.21037/ apm-20-1607

Conflicts of Interest: All authors have completed the ICMJE uniform disclosure form (available at http://dx.doi. org/10.21037/apm-20-1607). The authors have no conflicts of interest to declare.

Ethical Statement: The authors are accountable for all aspects of the work in ensuring that questions related to the accuracy or integrity of any part of the work are appropriately investigated and resolved. The study was conducted in accordance with the Declaration of Helsinki (as revised in 2013). The study was approved by institutional ethics board of Juntendo University Hospital (No. 18-316) and individual consent for this retrospective analysis was waived.

Open Access Statement: This is an Open Access article distributed in accordance with the Creative Commons Attribution-NonCommercial-NoDerivs 4.0 International License (CC BY-NC-ND 4.0), which permits the noncommercial replication and distribution of the article with the strict proviso that no changes or edits are made and the original work is properly cited (including links to both the formal publication through the relevant DOI and the license). See: https://creativecommons.org/licenses/by-nc-nd/4.0/.

\section{References}

1. Wei S, Chen M, Chen N, et al. Feasibility and safety of robot-assisted thoracic surgery for lung lobectomy in patients with non-small cell lung cancer: a systematic review and meta-analysis. World J Surg Oncol 2017;15:98.

2. Kernstine KH. Robotics in thoracic surgery. Am J Surg 2004;188:89S-97S.

3. Park BJ, Flores RM, Rusch VW. Robotic assistance for video-assisted thoracic surgical lobectomy: technique and initial results. J Thorac Cardiovasc Surg 2006;131:54-9. 
4. Augustin F, Bodner J, Wykypiel H, et al. Initial experience with robotic lung lobectomy: report of two different approaches. Surg Endosc 2011;25:108-13.

5. Yan TD, Black D, Bannon PG, et al. Systematic review and meta-analysis of randomized and nonrandomized trials on safety and efficacy of video assisted thoracic surgery lobectomy for early-stage non-small-cell lung cancer. J Clin Oncol 2009;27:2553-62.

6. Cao C, Manganas C, Ang SC, et al. A meta-analysis of unmatched and matched patients comparing videoassisted thoracoscopic lobectomy and conventional open lobectomy. Ann Cardiothorac Surg 2012;1:16-23.

7. Oh DS, Reddy RM, Gorrepati ML, et al. Robotic-Assisted, Video-Assisted Thoracoscopic and Open Lobectomy: Propensity-Matched Analysis of Recent Premier Data. Ann Thorac Surg 2017;104:1733-40.

8. Mahieu J, Rinieri P, Bubenheim M, et al. RobotAssisted Thoracoscopic Surgery versus Video-Assisted Thoracoscopic Surgery for Lung Lobectomy: Can a Robotic Approach Improve Short-Term Outcomes and Operative Safety? Thorac Cardiovasc Surg 2016;64:354-62.

9. Veronesi G, Galetta D, Maisonneuve P, et al. Four-arm robotic lobectomy for the treatment of early-stage lung cancer. J Thorac Cardiovasc Surg 2010;140:19-25.

10. Flores RM. Video-Assisted Thoracic Surgery (VATS) Lobectomy: Focus on Technique. World J Surg 2010;34:616-20.

11. Lewis RJ. Simultaneously stapled lobectomy: a safe technique for video-assisted thoracic surgery. J Thorac Cardiovasc Surg 1995;109:619-25.

12. Toker A, KabaE, Ayalp K, et al, Robotic lung resections: video-assisted thoracic surgery based approach. J Vis Surg 2017;3:15.

13. Lee J, Lee JY, Choi JS, et al. Comparison of Uniportal versus Multiportal Video-Assisted Thoracoscopic Surgery Pulmonary Segmentectomy. Korean J Thorac Cardiovasc Surg 2019;52:141-7.

14. Darr C, Cheufou D, Weinreich G, et al. Robotic thoracic surgery results in shorter hospital stay and lower postoperative pain compared to open thoracotomy: a matched pairs analysis. Surg Endosc 2017;31:4126-30.

15. Kwon ST, Zhao L, Reddy RM, et al. Evaluation of acute and chronic pain outcomes after robotic video-assisted thoracoscopic surgery, or open anatomic pulmonary resection. J Thorac Cardiovasc Surg 2017;154:652-59.e1.

16. Hirata I, Iwamoto M, Matsui H, et al. Droperidol Reduces Postoperative Nausea and Vomiting and Supports the
Continuation of Intravenous Patient-Controlled Analgesia with Fentanyl. J Pharm Pharm Sci 2020;23:220-30.

17. Nakata K, Mammoto T, Kita T, et al. Continuous epidural, not intravenous, droperidol inhibits pruritus, nausea, and vomiting during epidural morphine analgesia. J Clin Anesth 2002;14:121-5.

18. Joshi GP, Bonnet F, Shah R, et al. A systematic review of randomized trials evaluating regional techniques for post thoracotomy analgesia. Anesth Analg 2008;107:1026-40.

19. Meierhenrich R, Hock D, Kühn S, et al. Analgesia and pulmonary function after lung surgery: is a single intercostal nerve block plus patient-controlled intravenous morphine as effective as patient-controlled epidural anaesthesia? A randomized non-inferiority clinical trial. Br J Anaesth 2011;106:580-9.

20. Hegarty D. Post Thoracotomy Pain Syndrome: What Pain Management Options do we have? J Surg Transplant Sci 2017;5:1059.

21. Davies RG, Myles PS, Graham JM. A comparison of the analgesic efficacy and side-effects of paravertebral vs. epidural blockade for thoracotomy--a systematic review and meta-analysis of randomized trials. Br J Anaesth 2006;96:418-26.

22. Yeung JH, Gates S, Naidu BV, et al. Paravertebral block versus thoracic epidural for patients undergoing thoracotomy. Cochrane Database Syst Rev 2016;2:CD009121.

23. Yeap YL, Wolfe JW, Backfish-White KM, et al. Randomized Prospective Study Evaluating SingleInjection Paravertebral Block, Paravertebral Catheter, and Thoracic Epidural Catheter for Postoperative Regional Analgesia After Video-Assisted Thoracoscopic Surgery. J Cardiothorac Vasc Anesth 2020;34:1870-76.

24. Novellis P, Alloisio M, Vanni E, et al. Robotic lung cancer surgery: review of experience and costs. J Vis Surg 2017;3:39.

25. Lee JR. Anesthetic considerations for robotic surgery. Korean J Anesthesiol 2014;66:3-11.

26. Grider JS, Mullet TW, Saha SP, et al. A Randomized, Double-Blind Trial Comparing Continuous Thoracic Epidural Bupivacaine With and Without Opioid in Contrast to a Continuous Paravertebral Infusion of Bupivacaine for Post-thoracotomy Pain. J Cardiothorac Vasc Anesth, 2012;26:83-9.

27. Bromage PR, Camporesi EM, Durant PA, et al. Rostral spread of epidural morphine 1982;56:431-6.

28. Negre I, Gueneron JP, Ecoffey C, et al. Ventilatory response to carbon dioxide after intramuscular and 
epidural fentanyl. Anesth Analg 1987;66:707-10.

29. Obara S, Egan T. Pharmacokinetic and Pharmacodynamic Principles for Intravenous Anesthetics. In: Hemmings H, Egan T (eds). Pharmacokinetic and Pharmacodynamic (2nd edition). Amsterdam, Holland: Elsevier, 2019:20-43.

Cite this article as: Kawagoe I, Hayashida M, Satoh D, Kochiyama T, Fukuda M, Kishii J. Postoperative analgesia in patients undergoing robot-assisted thoracic surgery: a comparison between thoracic epidural analgesia and intercostal nerve block combined with intravenous patient-controlled analgesia. Ann Palliat Med 2021;10(2):1985-1993. doi: 10.21037/ apm-20-1607
30. Lin Y, Liu SS. Local anesthetics. In: Barash PG, Cullen BF, Stoelting RK, et al (eds). Clinical Anesthesia (7th edition). Philadelphia, USA: Lippincott Williams \& Wilkins, 2013:561-79. 